\title{
Daratumumab monotherapy in patients with treatment-refractory multiple myeloma (SIRIUS): an open-label, randomised, phase 2 trial
}

\begin{abstract}
Sagar Lonial, Brendan M Weiss, Saad Z Usmani, Seema Singhal, Ajai Chari, NizarJ Bahlis, Andrew Belch, Amrita Krishnan, Robert A Vescio, Maria Victoria Mateos, Amitabha Mazumder, Robert Z Orlowski, HeatherJ Sutherland, Joan Bladé, Emma C Scott, Albert Oriol, Jesus Berdeja, Mecide Gharibo, Don A Stevens, Richard LeBlanc, Michael Sebag, Natalie Callander, Andrzej Jakubowiak, Darrell White, Javier de la Rubia, Paul G Richardson, Steen Lisby, Huaibao Feng, Clarissa M Uhlar, Imran Khan, Tahamtan Ahmadi, Peter M Voorhees
\end{abstract}

\section{Summary}

Background New treatment options are needed for patients with multiple myeloma that is refractory to proteasome inhibitors and immunomodulatory drugs. We assessed daratumumab, a novel CD38-targeted monoclonal antibody, in patients with refractory multiple myeloma.

Methods In this open-label, multicentre, phase 2 trial done in Canada, Spain, and the USA, patients (age $\geq 18$ years) with multiple myeloma who were previously treated with at least three lines of therapy (including proteasome inhibitors and immunomodulatory drugs), or were refractory to both proteasome inhibitors and immunomodulatory drugs, were randomly allocated in a $1: 1$ ratio to receive intravenous daratumumab $8 \mathrm{mg} / \mathrm{kg}$ or $16 \mathrm{mg} / \mathrm{kg}$ in part 1 stage 1 of the study, to decide the dose for further assessment in part 2. Patients received $8 \mathrm{mg} / \mathrm{kg}$ every 4 weeks, or $16 \mathrm{mg} / \mathrm{kg}$ per week for 8 weeks (cycles 1 and 2), then every 2 weeks for 16 weeks (cycles 3-6), and then every 4 weeks thereafter (cycle 7 and higher). The allocation schedule was computer-generated and randomisation, with permuted blocks, was done centrally with an interactive web response system. In part 1 stage 2 and part 2 , patients received $16 \mathrm{mg} / \mathrm{kg}$ dosed as in part 1 stage 1 . The primary endpoint was overall response rate (partial response $[P R]+$ very good $P R+$ complete response $[C R]+$ stringent $C R)$. All patients who received at least one dose of daratumumab were included in the analysis. The trial is registered with ClinicalTrials.gov, number NCT01985126.

Findings The study is ongoing. In part 1 stage 1 of the study, 18 patients were randomly allocated to the $8 \mathrm{mg} / \mathrm{kg}$ group and 16 to the $16 \mathrm{mg} / \mathrm{kg}$ group. Findings are reported for the 106 patients who received daratumumab $16 \mathrm{mg} / \mathrm{kg}$ in parts 1 and 2. Patients received a median of five previous lines of therapy (range 2-14). 85 (80\%) patients had previously received autologous stem cell transplantation, $101(95 \%)$ were refractory to the most recent proteasome inhibitors and immunomodulatory drugs used, and $103(97 \%)$ were refractory to the last line of therapy. Overall responses were noted in 31 patients $(29 \cdot 2 \%, 95 \%$ CI $20 \cdot 8-38 \cdot 9)$-three $(2 \cdot 8 \%, 0 \cdot 6-8 \cdot 0)$ had a stringent CR, ten $(9 \cdot 4 \%, 4 \cdot 6-16 \cdot 7)$ had a very good PR, and $18(17 \cdot 0 \%, 10 \cdot 4-25 \cdot 5)$ had a PR. The median time to first response was 1.0 month (range 0.9-5.6). Median duration of response was 7.4 months (95\% CI 5.5-not estimable) and progression-free survival was $3 \cdot 7$ months (95\% CI 2-8-4 -6). The 12-month overall survival was $64 \cdot 8 \%(95 \%$ CI $51 \cdot 2-75 \cdot 5)$ and, at a subsequent cutoff, median overall survival was 17.5 months $(95 \%$ CI 13.7-not estimable). Daratumumab was well tolerated; fatigue (42 [40\%] patients) and anaemia (35 [33\%]) of any grade were the most common adverse events. No drug-related adverse events led to treatment discontinuation.

Interpretation Daratumumab monotherapy showed encouraging efficacy in heavily pretreated and refractory patients with multiple myeloma, with a favourable safety profile in this population of patients.

Funding Janssen Research \& Development.

\section{Introduction}

Multiple myeloma is a malignant plasma cell disorder that is characterised by bone, renal, haematological, and infectious complications due to accumulation of clonal plasma cells in the bone marrow and pathogenic antibody production. ${ }^{1}$ Although survival has improved substantially with new drug classes (eg, proteasome inhibitors and immunomodulatory drugs), along with autologous stem cell transplantation, most patients will die from refractory disease. .,3 $^{2,}$ Outcomes for patients who are resistant to proteasome inhibitors (bortezomib and carfilzomib) and immunomodulatory drugs (lenalidomide, thalidomide, and pomalidomide) are especially poor. Before the availability of carfilzomib and pomalidomide, median expected overall survival in these patients was 9 months. ${ }^{4}$ Additional treatment can be complicated by cytopenias, secondary to poor haematological reserves, and comorbidities such as renal insufficiency. Therefore, effective treatments that target novel pathways with little toxicity and favourable tolerability are needed. Monoclonal antibodies are a novel class of agents in myeloma, targeting cell surface
Published Online January 6, 2016 http://dx.doi.org/10.1016 S0140-6736(15)01120-4 See Online/Comment http://dx.doi.org/10.1016/ S0140-6736(15)01226-X

Department of Hematology and Medical Oncology, Winship Cancer Institute, Emory University, Atlanta, GA, USA (Prof S Lonial MD); Division of Hematology-Oncology, Department of Medicine, Abramson Cancer Center and Perelman School of Medicine, University of Pennsylvania, Philadelphia, PA, USA (Prof B M Weiss MD); Levine Cancer Institute/Carolinas Healthcare System, Charlotte, NC, USA (Prof S ZUsmani MD); Robert H Lurie Comprehensive Cancer Center, Division of Hematology/Oncology, Northwestern University Feinberg School of Medicine, Chicago, IL, USA (Prof S Singhal MD); Tisch Cancer Institute, Mount Sinai School of Medicine, New York NY, USA (Prof A Chari MD); Tom Baker Cancer Center, University of Calgary, Calgary, $A B$, Canada (Prof N J Bahlis MD); Cross Cancer Institute,

Edmonton, AB, Canad (Prof A Belch MD); The Judy and Bernard Briskin Myeloma Center, City of Hope, Duarte, CA, USA (Prof A Krishnan MD); Cedars-Sinai Outpatient Cancer Center at the Samuel Oschin Comprehensive Cancer Institute, Los Angeles, CA, USA (Prof R A Vescio MD); University Hospital of Salamanca/ Instituto de Investigación Biomédica de Salamanca, Salamanca, Spain (Prof M V Mateos MD); Perlmutter Cancer Center, NYU Langone Medical Center, New York, NY, USA (Prof A Mazumder MD); 
Department of Lymphoma and Myeloma, University of Texas MD Anderson Cancer Center, Houston, TX, USA (Prof R Z Orlowski MD); Leukemia/Bone Marrow Transplant Program, University of British Columbia, Vancouver, BC, Canada (Prof HJ Sutherland MD); Institut d'Investigacions Biomèdiques August $\mathrm{Pi}$ Sunyer, Hospital Clinic de Barcelona, Barcelona, Spain (ProfJ Bladé MD); Knight Cancer Institute, Oregon Health and Science University, Portland, OR, USA (Prof E ( Scott MD); Institut Català d'Oncologia, Institut Josep Carreras, Hospital Germans Trias i Pujol,

Barcelona, Spain (A Oriol MD); Sarah Cannon Research Institute, Nashville, TN, USA (J Berdeja MD); Rutgers Cancer Institute of New Jersey, Robert Wood Johnson Medical School, Rutgers, State University of New Jersey, New Brunswick, NJ, USA (Prof M Gharibo MD); Norton Health Care, Louisville, KY, USA (D A Stevens MD); Hôpital Maisonneuve-Rosemont, Montreal, QC, Canada (Prof R LeBlanc MD); Royal

Victoria Hospital, Montreal, QC, Canada (Prof M Sebag MD). University of Wisconsin

Medical School, Madison, WI,

USA (Prof N Callander MD); University of Chicago

Medicine, Chicago, IL, USA (Prof A Jakubowiak MD); Dalhousie University and Queen Elizabeth II Health

Sciences Center, Halifax, NS, Canada (Prof D White MD); Hospital Dr Peset and Universidad Católica

San Vicente Mártir, Valencia,

Spain (Prof) de la Rubia MD); Dana-Farber Cancer Institute, Harvard Medical School, Boston, MA, USA (Prof P G Richardson MD); Genmab, Copenhagen,

Denmark (S Lisby MD); Janssen Research and Development, Raritan, NJ, USA (H Feng PhD, I Khan MD); Janssen Research and Development, Spring House, PA, USA (C M Uhlar PhD, T Ahmadi MD); and Division of Hematology and Oncology, Lineberger Comprehensive Cancer Center, University of

North Carolina at Chapel Hill, Chapel Hill, NC, USA (Prof P M Voorhees MD)

\section{Research in context}

\section{Evidence before this study}

We searched PubMed on July 29, 2015, with no date restriction using the keywords "progression risk", "overall survival", "multiple myeloma", "relapsed", and "refractory". From the 45 articles identified, the evidence indicates that despite the introduction of new agents that have prolonged survival, multiple myeloma remains incurable because most patients relapse or become refractory to available treatments. Daratumumab, a human monoclonal antibody that binds CD38-expressing malignant cells, gained US Food and Drug Administration breakthrough therapy designation based on phase 1 data from a first-in-human study in patients with multiple myeloma who relapsed or were refractory to at least two previous therapies. The first-in-human study was expanded and we concurrently initiated our study to further investigate the selected dose schedule.

\section{Added value of this study}

The current study is the largest study so far of the single-agent activity of daratumumab $16 \mathrm{mg} / \mathrm{kg}$ in heavily pretreated patients with multiple myeloma who were refractory to both a proteasome inhibitor and an immunomodulatory drug. The overall response rate was 29\% and responses were rapid, deep, and durable. Efficacy was consistent in subgroups based on previous therapy and patients' characteristics, including age and renal function. Side-effects of daratumumab were clinically manageable, and no patient discontinued treatment because of drug-related adverse events. These data are in accord with results from the expansion of the first-in-human study in which the overall response rate was $36 \%$ in patients given daratumumab $16 \mathrm{mg} / \mathrm{kg}$ monotherapy.

\section{Implications of all the available evidence}

As a result of this study, daratumumab was the first monoclonal antibody approved by the US Food and Drug Administration for the treatment of refractory myeloma. Daratumumab is indicated for patients who have received at least three previous lines of therapy, including a proteasome inhibitor and an immunomodulatory drug, or who are double refractory to a proteasome inhibitor and an immunomodulatory drug. Based on its efficacy, with rapid, deep, and durable responses, and its favourable safety profile, further activity in combination regimens is being investigated. markers, such as SLAMF7 (CS-1) and CD38, with few off-target effects. ${ }^{5}$

Daratumumab is a first-in-class, human IgG1 monoclonal antibody that binds CD38-expressing malignant cells with high affinity and induces tumour cell death through diverse mechanisms of action, which include complement-dependent cytotoxicity, ${ }^{6,7}$ antibodydependent cell-mediated cytotoxicity, ${ }^{6,7}$ antibody-dependent cellular phagocytosis, ${ }^{7.8}$ and induction of apoptosis. ${ }^{7.9}$ In a first-in-human phase $1 / 2$ study of daratumumab monotherapy $(0 \cdot 005-24 \mathrm{mg} / \mathrm{kg})$ in patients with relapsed or relapsed and refractory multiple myeloma, a maximum tolerated dose was not achieved. ${ }^{10}$ In an expansion cohort of this study, the overall response rate (ORR) was 36\% with daratumumab $16 \mathrm{mg} / \mathrm{kg} .{ }^{10}$

In the current phase 2 study, we assessed daratumumab in patients with multiple myeloma and an unmet medical need - specifically, those who were refractory to their most recent treatment regimen after receiving at least three previous lines of therapy (including proteasome inhibitors and immunomodulatory drugs), or whose disease was refractory to both the most recent proteasome inhibitors and immunomodulatory drugs they had received, irrespective of the number of previous lines of treatment.

\section{Methods \\ Study design and participants}

This two-part, open-label, multicentre, phase 2 study started on Sept 30, 2013, at 26 sites in Canada, Spain, and the USA, and is ongoing. Inclusion criteria for patients included age at least 18 years old, documented secretory multiple myeloma, and evidence of disease progression on or within 60 days of the last dose of the most recent previous treatment regimen, based on the International Myeloma Working Group criteria. ${ }^{11,12}$ Eligible patients had responded to at least one previous treatment regimen, received an alkylating agent alone or in combination with other myeloma treatments, received at least three previous lines of treatment that included a proteasome inhibitor and an immunomodulatory drug, or had disease double refractory to the most recent proteasome inhibitor and immunomodulatory drug they had received, and had an Eastern Cooperative Oncology Group (ECOG) performance status score of 2 or lower.

Exclusion criteria included any antimyeloma treatment within 2 weeks, or autologous stem cell transplantation within 12 weeks, of day 1 of cycle 1 , and meningeal involvement of multiple myeloma and other malignancies within 5 years. Patients with absolute neutrophil counts of $1 \times 10^{9}$ per L or lower, haemoglobin concentration of $75 \mathrm{~g} / \mathrm{L}$ or lower, platelet counts of less than $50 \times 10^{9}$ per $\mathrm{L}$, and creatinine clearance of $20 \mathrm{~mL} / \mathrm{min}$ per $1.73 \mathrm{~mm}^{2}$ or lower were excluded. Other exclusion criteria were myocardial infarction within 1 year, uncontrolled or unstable angina, congestive heart failure (New York Heart Association Class III or IV), arrhythmia (grade 2 or higher), QTcF interval that was longer than $470 \mathrm{~ms}$, chronic obstructive pulmonary disease, persistent asthma, or a history of asthma within 5 years.

Ethics committees or institutional review boards at the study sites approved the study, which was done in 
accordance with the principles of the Declaration of Helsinki, the International Conference on Harmonisation, and the guidelines for Good Clinical Practice. All patients provided written informed consent.

\section{Randomisation and masking}

In part 1 stage 1 of the trial, patients were randomly allocated in a 1:1 ratio to the daratumumab $8 \mathrm{mg} / \mathrm{kg}$ group or $16 \mathrm{mg} / \mathrm{kg}$ group ( $\leq 20$ patients per group). Central randomisation was done with an interactive web response system, and patients were randomly allocated to treatment with a computer-generated randomisation schedule prepared under the supervision of the vendor. Randomisation was balanced by use of permuted blocks, and stratification factors included International Staging System staging (I, II, or III) and refractory status (ie, refractory to either a proteasome inhibitor or an immunomodulatory drug, refractory to both a proteasome inhibitor and immunomodulatory drug, or not refractory to either). Refractory was defined as disease progression on or within 60 days of the last dose. No one was masked to treatment assignment.

\section{Procedures}

A dose of daratumumab could be discontinued if the dose was deemed to be ineffective (ie, did not meet specified criteria for continuation) or poorly tolerated, or both, based on the results from the first interim analysis, which was done about 8 weeks after the last patient was enrolled in part 1 stage 1 of the study. After the first interim analysis, patients treated with an ineffective or poorly tolerated dose could crossover to the more effective dose if it was in their best interest according to the investigator treating them. A second interim analysis was done after another 25 patients were treated for at least 8 weeks in stage 2 of part 1 . In part 2 of the study, an expansion cohort of 65 patients was treated at the selected dose to assess safety and efficacy. Dose reductions were not permitted. Patients were treated until disease progression or unacceptable toxicity, and long-term follow-up began after treatment discontinuation. The planned end of the study at 18 months after the last patient received the first dose of daratumumab had not been reached. The results of a prespecified efficacy analysis about 6 months after the last patient received his or her first dose of daratumumab are presented.

Patients received one of two regimens of daratumumab intravenously: daratumumab at $16 \mathrm{mg} / \mathrm{kg}$ per week for 8 weeks (cycles 1 and 2), then every 2 weeks for 16 weeks (cycles 3-6), then every 4 weeks thereafter (cycle 7 and higher); or daratumumab $8 \mathrm{mg} / \mathrm{kg}$ every 4 weeks continuously. First infusions of daratumumab were $1000 \mathrm{~mL}$ at $50 \mathrm{~mL} / \mathrm{h}$. If no infusion-related reactions occurred, the dose was increased in $50 \mathrm{~mL} / \mathrm{h}$ increments to $200 \mathrm{~mL} / \mathrm{h}$. Second infusions were $500 \mathrm{~mL}$ at $50 \mathrm{~mL} / \mathrm{h}$ and increased to $200 \mathrm{~mL} / \mathrm{h}$. Subsequent $500 \mathrm{~mL}$ infusions were at $100 \mathrm{~mL} / \mathrm{h}$ and increased to $200 \mathrm{~mL} / \mathrm{h}$.
Pre-infusion medications, $1 \mathrm{~h}( \pm 0 \cdot 25 \mathrm{~h})$ before dosing, were methylprednisolone (100 $\mathrm{mg}$ intravenously for the first and second infusions and $60 \mathrm{mg}$ thereafter), paracetamol (650-1000 mg), and diphenhydramine (25-50 mg) or equivalent antihistamine drug. Oral methylprednisolone $(20 \mathrm{mg})$ or equivalent was administered on the 2 days after all daratumumab infusions.

\section{Outcomes}

The primary endpoint was overall response rate (ORR; partial response $[\mathrm{PR}]+$ very good $\mathrm{PR}+$ complete response $[C R]+$ stringent $C R)$. Secondary endpoints included duration of response, progression-free survival (PFS), overall survival, and clinical benefit rate (minimal response+ORR). Response was confirmed on two consecutive measurements, and data were assessed by an independent review committee.

Safety assessments were the monitoring of adverse events, physical examinations, electrocardiogram monitoring, clinical laboratory measurements, vital sign

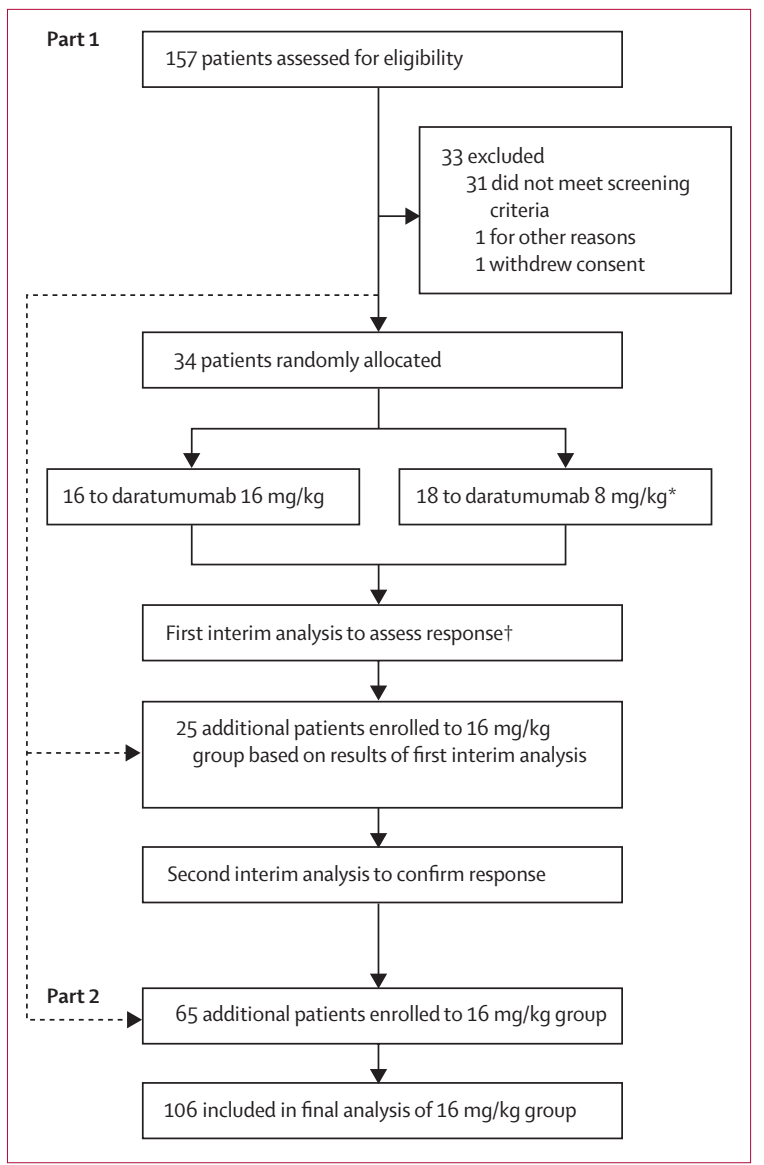

\section{Figure 1: Trial profile}

*Three patients were crossed over to the daratumumab $16 \mathrm{mg} / \mathrm{kg}$ group after the first interim analysis, but were not included in the efficacy analysis of the $16 \mathrm{mg} / \mathrm{kg}$ group. †Treatment with $8 \mathrm{mg} / \mathrm{kg}$ was discontinued because the overall response rate did not meet the prespecified criteria for continuation.
Correspondence to: Prof Sagar Lonial, Department of Hematology and Medical Oncology, Winship Cancer Institute, Emory University, 201 Dowman Drive, Atlanta, GA 30322, USA sloni01@emory.edu 


\begin{tabular}{|c|c|}
\hline & $\begin{array}{l}\text { Daratumumab } \\
16 \mathrm{mg} / \mathrm{kg}(\mathrm{n}=106)\end{array}$ \\
\hline \multicolumn{2}{|l|}{ Age (years) } \\
\hline Median (range) & $63 \cdot 5(31 \cdot 0-84 \cdot 0)$ \\
\hline 18 to $<65$ & $58(55 \%)$ \\
\hline 65 to $<75$ & $36(34 \%)$ \\
\hline$\geq 75$ & $12(11 \%)$ \\
\hline Men & $52(49 \%)$ \\
\hline \multicolumn{2}{|l|}{ Ethnic origin } \\
\hline White & $84(79 \%)$ \\
\hline Black or African American & $15(14 \%)$ \\
\hline Asian & $4(4 \%)$ \\
\hline Not reported, other, unknown & $3(3 \%)$ \\
\hline \multicolumn{2}{|l|}{ Eastern Cooperative Oncology Group score } \\
\hline 0 & $29(27 \%)$ \\
\hline 1 & $69(65 \%)$ \\
\hline 2 & $8(8 \%)$ \\
\hline \multicolumn{2}{|l|}{ International Staging System staging } \\
\hline 1 & $26(25 \%)$ \\
\hline ॥ & $40(38 \%)$ \\
\hline III & $40(38 \%)$ \\
\hline \multicolumn{2}{|l|}{ Cytogenetics profile* } \\
\hline $\mathrm{t}(4 ; 14)$ & $9(10 \%)$ \\
\hline del17p & $16(17 \%)$ \\
\hline del13q & $30(32 \%)$ \\
\hline amp1q21 & $23(24 \%)$ \\
\hline Other & $43(45 \%)$ \\
\hline \multicolumn{2}{|c|}{ Renal function (baseline creatinine clearance) } \\
\hline$\geq 1.0 \mathrm{~mL} / \mathrm{s}(\geq 60 \mathrm{~mL} / \mathrm{min})$ & $60(57 \%)$ \\
\hline 0.5 to $<1.0 \mathrm{~mL} / \mathrm{s}(30$ to $<60 \mathrm{~mL} / \mathrm{min})$ & $42(40 \%)$ \\
\hline$<0.5 \mathrm{~mL} / \mathrm{s}(<30 \mathrm{~mL} / \mathrm{min})$ & $4(4 \%)$ \\
\hline \multicolumn{2}{|l|}{ Extramedullary plasmacytomas } \\
\hline \multirow[t]{2}{*}{$\geq 1$} & $14(13 \%)$ \\
\hline & ntinues in nex \\
\hline
\end{tabular}

measurements, and ECOG performance status. Severity of adverse events was assessed with the National Cancer Institute Common Terminology Criteria for Adverse Events (version 4.03). ${ }^{13}$

\section{Statistical analysis}

Briefly, about 100 patients would be treated during the study for the selected dose schedule. Response assessments were done by an independent review committee using the International Myeloma Working Group response criteria. ${ }^{11,12}$ ORRs were reported with two-sided 95\% exact CI. Additionally, the number and percentage of patients in each response category were presented. Time-to-event endpoints, including duration of response, PFS, and overall survival, were analysed descriptively with the Kaplan-Meier method. All patients who received at least one dose of daratumumab were used for efficacy and safety analyses.

The study is registered with ClinicalTrials.gov, number NCT01985126.

\begin{tabular}{|c|c|}
\hline & $\begin{array}{l}\text { Daratumumab } \\
16 \mathrm{mg} / \mathrm{kg}(\mathrm{n}=106)\end{array}$ \\
\hline \multicolumn{2}{|l|}{ (Continued from previous column) } \\
\hline Time since initial diagnosis (years; median, range) & $4 \cdot 8(1 \cdot 1-23 \cdot 8)$ \\
\hline \multicolumn{2}{|l|}{ Lines of previous therapy } \\
\hline$>3$ & $87(82 \%)$ \\
\hline Median (range) & $5(2-14)$ \\
\hline Previous proteasome inhibitor & $106(100 \%)$ \\
\hline Bortezomib & $105(99 \%)$ \\
\hline Carfilzomib & $53(50 \%)$ \\
\hline Previous immunomodulatory drug & $106(100 \%)$ \\
\hline Lenalidomide & $105(99 \%)$ \\
\hline Pomalidomide & $67(63 \%)$ \\
\hline Thalidomide & $47(44 \%)$ \\
\hline Previous steroids & $106(100 \%)$ \\
\hline Dexamethasone & $106(100 \%)$ \\
\hline Previous autologous stem cell transplantation & $85(80 \%)$ \\
\hline \multicolumn{2}{|l|}{ Refractory to } \\
\hline $\begin{array}{l}\text { Both proteasome inhibitor and } \\
\text { immunomodulatory drug }\end{array}$ & $101(95 \%)$ \\
\hline Last line of previous therapy & $103(97 \%)$ \\
\hline Bortezomib & $95(90 \%)$ \\
\hline Carfilzomib & $51(48 \%)$ \\
\hline Lenalidomide & $93(88 \%)$ \\
\hline Pomalidomide & $67(63 \%)$ \\
\hline Thalidomide & $29(27 \%)$ \\
\hline Alkylating agent & $82(77 \%)$ \\
\hline Bortezomib + lenalidomide & $87(82 \%)$ \\
\hline Bortezomib + lenalidomide + carfilzomib & $42(40 \%)$ \\
\hline Bortezomib + lenalidomide + pomalidomide & $57(54 \%)$ \\
\hline $\begin{array}{l}\text { Bortezomib + lenalidomide + carfilzomib + } \\
\text { pomalidomide }\end{array}$ & $33(31 \%)$ \\
\hline \multicolumn{2}{|c|}{$\begin{array}{l}\text { Data are number }(\%) \text {, unless otherwise indicated. }{ }^{*} \text { Cytogenetic abnormalities } \\
\text { were detected by fluorescence in-situ hybridisation or karyotyping, or both at } \\
\text { baseline }(n=95) \text {. }\end{array}$} \\
\hline
\end{tabular}

\section{Role of the funding source}

The clinical investigators and funder were responsible for adherence to the study design and analysis plan. The investigators and their research teams gathered the data, and the funder compiled the data for summation and analysis and confirmed its accuracy. The funder coordinated the development of the manuscript and funded writing assistance. The authors had full access to the study data, participated in the development of the manuscript, and made the final decision to submit the manuscript for publication.

\section{Results}

124 patients received at least one dose of daratumumab (18 received $8 \mathrm{mg} / \mathrm{kg}$, 106 received $16 \mathrm{mg} / \mathrm{kg}$; figure 1). The clinical cutoff date for primary analysis was Jan 9, 2015, 7.7 months after the last patient had received the first dose. At the first interim analysis, the 
daratumumab $8 \mathrm{mg} / \mathrm{kg}$ group did not meet the criteria for expansion because of an ORR of $11.1 \%$ (95\% CI 1.4-34.7). Baseline characteristics of this group are presented in the appendix. Concurrent pharmacokinetic analyses of the $8 \mathrm{mg} / \mathrm{kg}$ dose in the first-in-human study indicated that drug concentrations were probably less than the trough threshold for target saturation. ${ }^{10}$ The results of these analyses also suggested that, although $24 \mathrm{mg} / \mathrm{kg}$ daratumumab would have been well tolerated, the gain in clinical benefit would have been small with this dose compared with $16 \mathrm{mg} / \mathrm{kg}$. Three patients in the $8 \mathrm{mg} / \mathrm{kg}$ group crossed over to the $16 \mathrm{mg} / \mathrm{kg}$ group, and were included in the $8 \mathrm{mg} / \mathrm{kg}$ group in all analyses. At the second interim analysis, after an additional 25 patients were treated in the $16 \mathrm{mg} / \mathrm{kg}$ group, the cumulative ORR justified expansion of the study into part 2 and an additional 65 patients were enrolled.

All 106 patients in the daratumumab $16 \mathrm{mg} / \mathrm{kg}$ group had been previously treated with proteasome inhibitors and immunomodulatory drugs (bortezomib 105 [99\%], carfilzomib 53 [50\%], lenalidomide 105 [99\%], pomalidomide 67 [63\%], and thalidomide 47 [44\%]; table 1). All patients had received dexamethasone previously, and $87(82 \%)$ patients had previously received more than three lines of therapy. Patients were highly refractory, with $103(97 \%)$ refractory to the last line of therapy before enrolment in the study and 101 (95\%) refractory to the most recent proteasome inhibitors and immunomodulatory drugs (table 1). Refractoriness to specific agents included alkylating agents (82 [77\%] patients), pomalidomide (67 [63\%] patients), carfilzomib (51 [48\%] patients), and bortezomib plus lenalidomide (double refractoriness, 87 [82\%] patients; table 1). 70 (66\%) patients were refractory to at least three of four drugs: bortezomib, lenalidomide, pomalidomide, and carfilzomib, and 33 (31\%) patients were refractory to all four agents. Median time since initial diagnosis was 4.8 years (range $1 \cdot 1-23 \cdot 8)$, median number of lines of previous therapy was five (range 2-14), and 85 (80\%) patients received previous autologous stem cell transplantation (table 1).

The median and mean numbers of treatment cycles given to patients were 4.0 (range 1-16) and $5 \cdot 3$ (SD 3.7), respectively, with 40 (38\%) patients receiving six or more cycles of daratumumab $16 \mathrm{mg} / \mathrm{kg}$. Median duration was $7.0 \mathrm{~h}$ (range $1.5-14 \cdot 3$ ) for the first infusion of daratumumab $(\mathrm{n}=106), 4 \cdot 2 \mathrm{~h}(2 \cdot 7-8 \cdot 5)$ for the second infusion $(n=103)$, and $3 \cdot 4 \mathrm{~h}(1 \cdot 1-6 \cdot 7)$ for subsequent infusions $(\mathrm{n}=1105) .90(85 \%)$ of 106 patients discontinued daratumumab $16 \mathrm{mg} / \mathrm{kg}$ : 82 (77\%) because of progressive disease, five (5\%) because of treatment-unrelated adverse events, and three (3\%) as a result of consent withdrawal because of symptoms related to disease progression.

In the daratumumab $16 \mathrm{mg} / \mathrm{kg}$ group, overall response was noted in 31 of 106 patients (ORR 29.2\%, 95\% CI 20.8-38.9) based on assessment by an independent review committee (figure 2; table 2 ). Three (3\%) patients

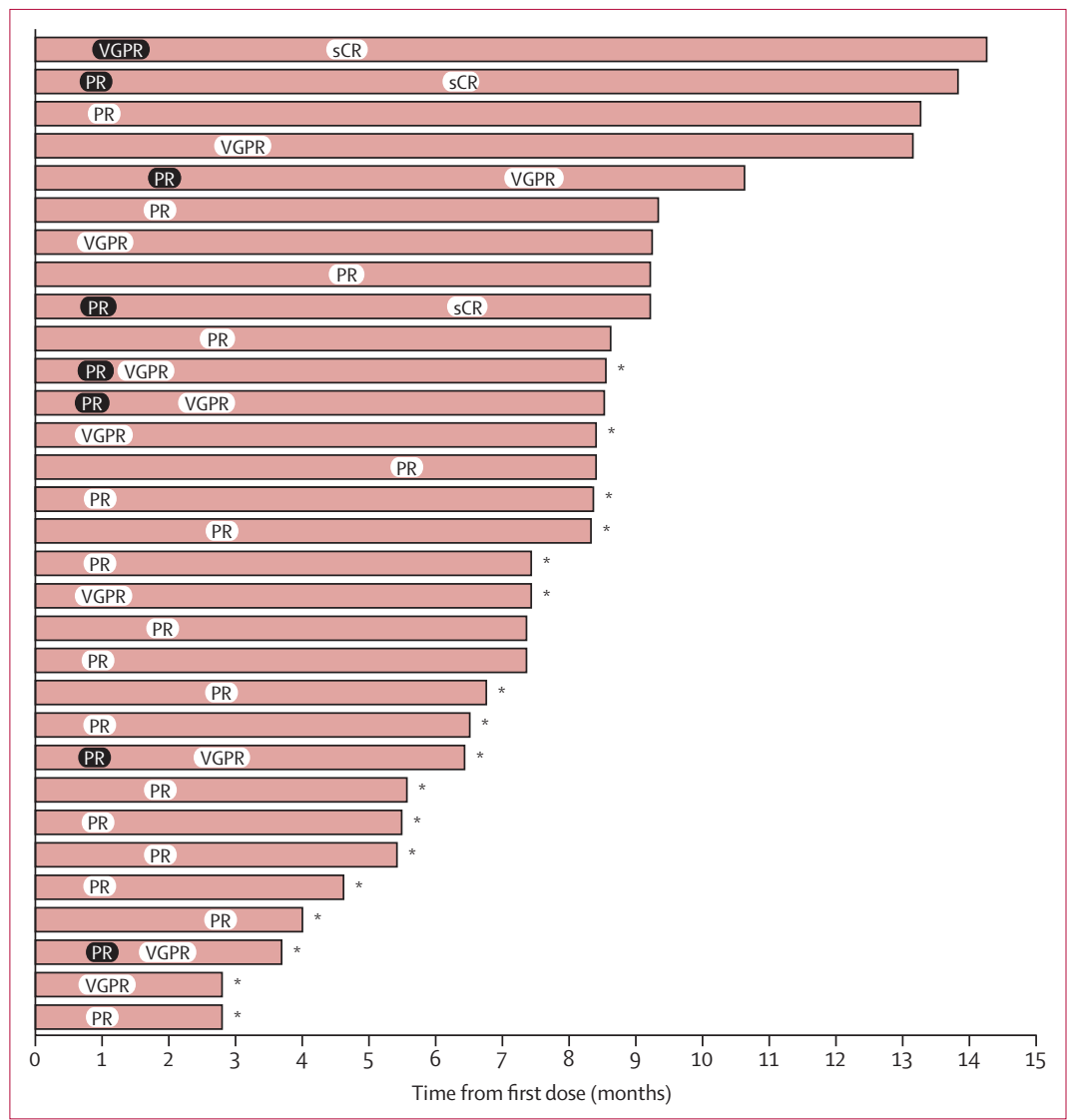

Figure 2: Swim-lane plot of responders in the daratumumab $16 \mathrm{mg} / \mathrm{kg}$ group

Black ovals indicate first response and white ovals indicate best response. VGPR=very good partial response. $\mathrm{s} C \mathrm{R}=$ stringent complete response. $\mathrm{PR}=$ partial response. *Disease progression.

\begin{tabular}{|c|c|}
\hline & $\begin{array}{l}\text { Daratumumab } 16 \mathrm{mg} / \mathrm{kg} \\
\text { group }(\mathrm{n}=106)\end{array}$ \\
\hline Stringent complete response & $3(2 \cdot 8 \%, 0 \cdot 6-8 \cdot 0)$ \\
\hline Complete response & 0 \\
\hline Very good partial response & $10(9 \cdot 4 \%, 4 \cdot 6-16 \cdot 7)$ \\
\hline Partial response & $18(17 \cdot 0 \%, 10 \cdot 4-25 \cdot 5)$ \\
\hline Minimal response & $5(4 \cdot 7 \%, 1 \cdot 5-10 \cdot 7)$ \\
\hline Stable disease & $46(43 \cdot 4 \%, 33 \cdot 8-53 \cdot 4)$ \\
\hline Progressive disease & $18(17 \cdot 0 \%, 10 \cdot 4-25 \cdot 5)$ \\
\hline Not evaluable & $6(5 \cdot 7 \%, 2 \cdot 1-11 \cdot 9)$ \\
\hline Overall response rate* & $31(29 \cdot 2 \%, 20 \cdot 8-38 \cdot 9)$ \\
\hline Clinical benefit rate $\dagger$ & $36(34 \cdot 0 \%, 25 \cdot 0-43 \cdot 8)$ \\
\hline Very good partial response or better $\neq$ & $13(12 \cdot 3 \%, 6 \cdot 7-20 \cdot 1)$ \\
\hline \multicolumn{2}{|c|}{$\begin{array}{l}\text { Data are number }(\%, 95 \% \mathrm{Cl}) \text {. }{ }^{*} \text { Defined as stringent complete response, complete } \\
\text { response, very good partial response, plus partial response. } † \text { Defined as overall } \\
\text { response rate plus minimal response. } \neq \text { Defined as stringent complete response, } \\
\text { complete response, plus very good partial response. }\end{array}$} \\
\hline
\end{tabular}

See Online for appendix

achieved stringent CR, ten (9\%) achieved very good PR, and 18 (17\%) patients had PR (table 2). The clinical benefit rate was $34 \cdot 0 \%(95 \%$ CI $25 \cdot 0-43 \cdot 8$; table 2$)$. The median time to first response was 1.0 month (range 
Figure 3: Overall response rate in patient subgroups in the daratumumab $16 \mathrm{mg} / \mathrm{kg}$ group Exact 95\% Cls are provided. $\mathrm{ORR}=$ overall response rate. ISS=International Staging System. IMWG=International Myeloma Working Group. $\mathrm{NE}=$ not estimable.

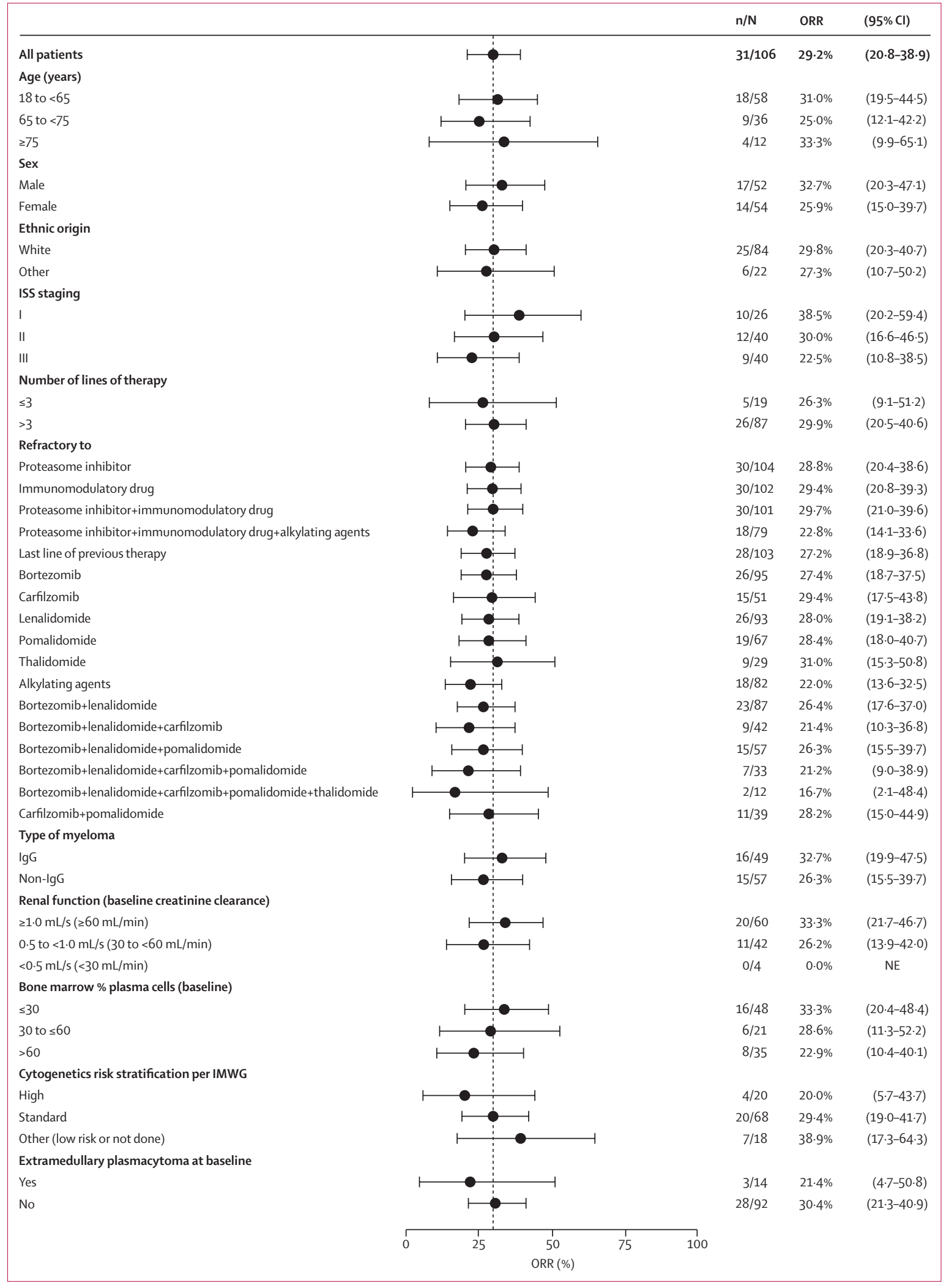


$0 \cdot 9-5 \cdot 6)$, and responses improved in eight $(25 \cdot 8 \%)$ of 31 patients over time (figure 2 ). Responses were noted in prespecified subgroups irrespective of previous lines of therapy and refractory status (figure 3 ). Overall responses were noted in 30 of 101 patients $(29 \cdot 7 \%, 21 \cdot 0-39 \cdot 6)$ who were refractory to both proteasome inhibitors and immunomodulatory drugs (figure 3), and in 20 of 70 patients $(28 \cdot 6 \%, 18 \cdot 4-40 \cdot 6)$ who were refractory to at least three of four agents (bortezomib, lenalidomide, carfilzomib, and pomalidomide). Of 12 patients who were refractory to five agents (bortezomib, lenalidomide, carfilzomib, pomalidomide, and thalidomide), two $(16 \cdot 7 \%, 2 \cdot 1-48 \cdot 4)$ achieved responses after daratumumab treatment. Overall responses occurred in the non-treatment-based subgroups: three of 14 patients $(21 \cdot 4 \%, 4 \cdot 7-50 \cdot 8)$ with extramedullary disease, four of 20 patients $(20 \%, 5 \cdot 7-43 \cdot 7)$ with baseline high-risk cytogenetics, 11 of 42 patients $(26 \cdot 2 \%, 13 \cdot 9-42 \cdot 0)$ with moderate renal impairment $(0 \cdot 5-1.0 \mathrm{~mL} / \mathrm{s}$ [30-60 $\mathrm{mL} / \mathrm{min}])$, and four of 12 patients $(33 \cdot 3 \%$, 9.9-65.1) older than 75 years (figure 3 ).

The median follow-up of patients was 9.3 months (range $0 \cdot 5-14 \cdot 4$ ), and median duration of response was 7.4 months (95\% CI 5.5-not estimable [NE]). Median PFS was 3.7 months (95\% CI 2.8-4.6; figure 4A), and the median overall survival was not reached $(13 \cdot 7-\mathrm{NE}$; figure 4B). The 12-month overall survival was $64.8 \%$ (95\% CI 51.2-75.5). Median overall survival was not reached in responders and was 13.7 months (95\% CI, 8.6-NE) in non-responders (appendix). 29 (94\%) of 31 responders treated with daratumumab $16 \mathrm{mg} / \mathrm{kg}$ were still alive, compared with $45(60 \%)$ of 75 non-responders. A subsequent clinical cutoff for a safety update to meet regulatory requirements was June 30, 2015. With this update, the median overall survival was 17.5 months $(13 \cdot 7-\mathrm{NE})$.

Daratumumab was well tolerated, and no patients discontinued because of drug-related treatment-emergent adverse events, infusion-related reactions, or death. The safety profile in the $8 \mathrm{mg} / \mathrm{kg}$ group was similar to that in the $16 \mathrm{mg} / \mathrm{kg}$ group (appendix). In the $16 \mathrm{mg} / \mathrm{kg}$ group, the most common haematological treatment-emergent adverse events of any grade $(\geq 20 \%)$ were anaemia (35 [33\%] patients), thrombocytopenia (27 [25\%]), and neutropenia (24 [23\%]; table 3). Grade 3 or higher anaemia and thrombocytopenia occurred more frequently in nonresponders (24 [32\%] and 18 [24\%] of 75 patients, respectively) than in responders (one [3\%] and two [6\%] of 31 patients, respectively). Grade 3 or higher neutropenia rates were similar in non-responders (nine [12\%]) and responders (four [13\%]). Few patients required additional supportive care: $40(38 \%)$ of 106 received red blood cell transfusions, $14(13 \%)$ had platelet transfusions, and eight $(8 \%)$ needed granulocyte colony-stimulating factor. Blood transfusions were unaffected by previously reported daratumumab interference with blood typing assays. ${ }^{14}$ Fatigue (42 [40\%] of 106 patients) and nausea (31 [29\%]

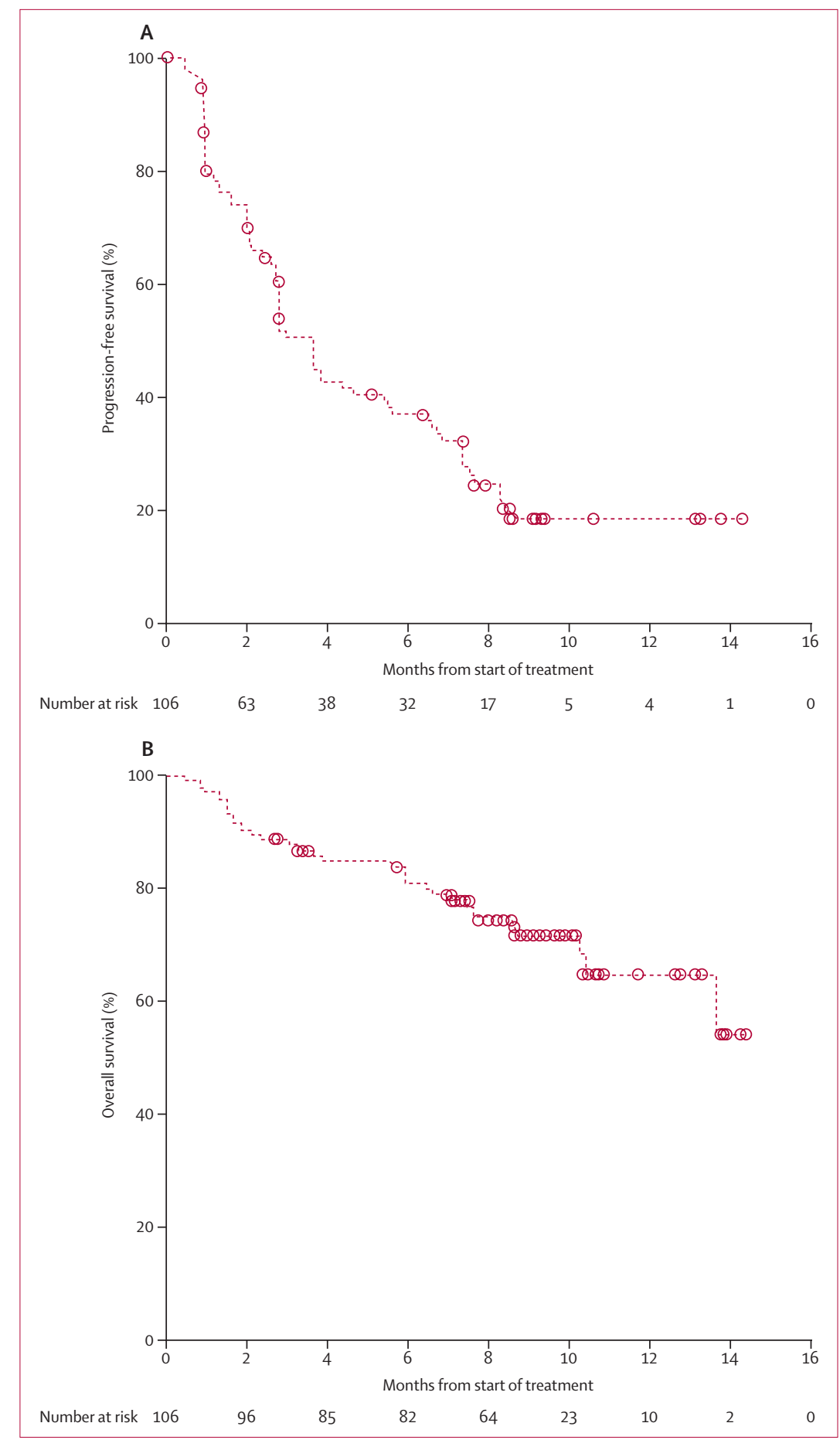

Figure 4: Progression-free survival (A) and overall survival (B) in the daratumumab $16 \mathrm{mg} / \mathrm{kg}$ group

patients) of any grade were the most prevalent nonhaematological treatment-emergent adverse events in the $16 \mathrm{mg} / \mathrm{kg}$ daratumumab group (table 3). 32 (30\%) patients had serious treatment-emergent adverse events, and $24(23 \%)$ had grade 3 or 4 serious treatment-emergent adverse events. Infusion-related reactions occurred in 


\begin{tabular}{|lcc|}
\hline & \multicolumn{2}{c|}{ Daratumumab $16 \mathbf{~ m g} / \mathbf{k g}(\mathbf{n}=106)$} \\
\cline { 2 - 3 } & Any grade & Grade 3 or 4 \\
\hline Fatigue & $42(40 \%)$ & $3(3 \%)$ \\
Anaemia & $35(33 \%)$ & $25(24 \%)$ \\
Nausea & $31(29 \%)$ & 0 \\
Thrombocytopenia & $27(25 \%)$ & $20(19 \%)$ \\
Neutropenia & $24(23 \%)$ & $13(12 \%)$ \\
Back pain & $23(22 \%)$ & $3(3 \%)$ \\
Cough & $22(21 \%)$ & $0(0 \%)$ \\
Data are number $(\%)$. & & \\
\hline Table 3: Most common $(\geq 20 \%)$ & treatment-emergent adverse events \\
\hline
\end{tabular}

$45(42 \%)$ patients and were predominantly grade 1 or 2 (grade 3 occurred in five [5\%] patients; no grade 4 infusionrelated reactions were reported). 39 (37\%) patients experienced infusion-related reactions during the first infusion and only six (6\%) patients had more than one infusion-related reaction. The most common ( $\geq 5 \%)$ infusion-related reactions included nasal congestion (13 [12\%]), throat irritation (seven [7\%]), and cough, dyspnoea, chills, and vomiting (six [6\%] each; appendix). No patient discontinued daratumumab because of an infusion-related reaction. No immunogenicity was reported.

Five (5\%) patients discontinued treatment because of treatment-emergent adverse events (two with general physical health deterioration (ie, progressive disease), and one each with H1N1 influenza, hypercalcaemia, and spinal cord compression). 31 (29\%) patients died after treatment with daratumumab 16 mg/kg: 29 (27\%) because of progressive disease and two (2\%) because of adverse events (cardiorespiratory failure secondary to H1N1 influenza complications, and general health deterioration secondary to complications of aspiration pneumonia).

\section{Discussion}

Daratumumab monotherapy showed substantial clinical activity, with an ORR of $29 \%$, and was well tolerated in patients with multiple myeloma who had been heavily treated; most patients were double refractory to bortezomib and lenalidomide, and many were refractory to pomalidomide or carfilzomib. Resistance to any previous therapy had no effect on the activity of daratumumab, lending support to a novel mechanism of action, but these findings need to be confirmed in larger studies. Similar response rates were also noted in patients with moderate renal impairment, older than 75 years of age, and with extramedullary disease or high-risk baseline cytogenetic characteristics. ${ }^{15}$ Although this study did not have a control arm, patients with the degree of treatment refractoriness in our study historically have poor outcomes. ${ }^{4}$ Follow-up is ongoing and thus more complete survival data will become available.
The results of our study corroborate the previously reported efficacy of daratumumab $16 \mathrm{mg} / \mathrm{kg}$ monotherapy in relapsed or refractory patients with multiple myeloma. ${ }^{10}$ Elotuzumab, a monoclonal antibody in development that targets SLAMF7, ${ }^{5}$ but lacks single-agent activity, ${ }^{16}$ increased median PFS by 4.5 months compared with the control arm in combination with lenalidomide and dexamethasone in a population of patients who were less heavily pretreated than those in the our study. ${ }^{17}$

Deep responses of very good PR or better, particularly stringent CR, are associated with improved long-term outcomes in patients with newly diagnosed multiple myeloma. ${ }^{18,19}$ Whether the same trend occurs in patients with relapsed and refractory multiple myeloma remains to be seen, although many patients treated in our study had responses to daratumumab that improved over time and might contribute to prolonged overall survival. These high-quality responses (9\% very good PR, 3\% stringent CR) are notable in treatment-refractory patients with multiple myeloma. The rate of high-quality responses with single-agent daratumumab were higher than those with pomalidomide monotherapy in the $\mathrm{mm}$ 002 clinical trial ( $2 \%$ of patients with at least a very good PR). ${ }^{20} 61 \%$ of 108 patients in the pomalidomide monotherapy group were refractory to lenalidomide and bortezomib, and had received a median of five previous lines of therapy. In the PX-171-003-A1 study ${ }^{21}$ of carfilzomib monotherapy in 266 patients, responses of very good PR or better were noted in $6 \%$ of patients. Similar to the present study and $\mathrm{mm}-002,{ }^{20}$ the patients were heavily pretreated with a median of five lines of therapy and $64 \%$ were refractory to lenalidomide and bortezomib. Peri-infusion doses of corticosteroids were used in our study to manage infusion-related reactions, and were thought to be substantially lower than the therapeutic use of dexamethasone in earlier lines of therapies to treat multiple myeloma. Thus, daratumumab can be regarded as a monotherapy in these patients who were refractory to dexamethasone.

When considering daratumumab monotherapy for treatment of refractory multiple myeloma, it should be placed in the context of other combination regimens that are in use in these patients. In the pomalidomide alone versus pomalidomide plus low-dose dexamethasone treatment groups of the mm-002 phase 2 study, $61 \%$ and $62 \%$ of 108 and 113 patients, respectively, were refractory to both bortezomib and lenalidomide, and in these double-refractory patients the ORR was $21 \%$ and $31 \%$, median PFS was 2.0 and 3.8 months, median duration of response was 11.4 and 6.5 months, and median overall survival was $12 \cdot 5$ and 13.4 months, respectively. ${ }^{20}$ In the PX-171-003-A1 study, in those treated with carfilzomib monotherapy who were refractory to both bortezomib and lenalidomide, the ORR was $15 \%$, median duration of response was 7.8 months, and median overall survival was 11.9 months. ${ }^{21}$ Thus, the ORR of $29 \%$, median PFS of 3.7 months, and median 
duration of response of 7.4 months in our ongoing study of daratumumab monotherapy compare favourably with these agents, particularly because most of the patients were refractory to both proteasome inhibitors and immunomodulatory drugs $(95 \%)$, and many were refractory to pomalidomide (63\%) or carfilzomib (48\%; table 1).

Daratumumab has a favourable safety profile compared with other available agents, and results in clinically manageable side-effects. No patient treated with $16 \mathrm{mg} / \mathrm{kg}$ discontinued daratumumab treatment because of a treatment-related adverse event. This result compares favourably with the substantial risk of neutropenia, febrile neutropenia, and infections with pomalidomide and dexamethasone reported in other studies. Additionally, non-haematological toxicities, such as cardiopulmonary and renal side-effects, are an important consideration with carfilzomib, particularly in patients with advanced disease. ${ }^{21,22}$ Lower rates of neutropenia were reported with single-agent daratumumab, and patients only routinely received steroids during the peri-infusion period and there was low use of granulocyte colony stimulating factor. Infusion-related reactions were easily managed, were usually grade 1 or 2 , and did not lead to discontinuation. The overall favourable toxicity profile of daratumumab makes it an attractive drug for use in combination regimens, and it has shown early promising activity in combination with lenalidomide and dexamethasone. ${ }^{23}$

In conclusion, daratumumab seems to be an effective option for patients with relapsed and refractory multiple myeloma for whom available treatments have been exhausted. Based on deep and durable responses and a favourable safety profile, daratumumab $16 \mathrm{mg} / \mathrm{kg}$ seems suitable for treatment of patients with multiple myeloma. The tolerability of daratumumab in combination with other backbone agents is being assessed in early phase studies. ${ }^{24}$ Patients with early to late stages of multiple myeloma are being enrolled in randomised phase 3 studies of daratumumab in combination with bortezomib, or lenalidomide and dexamethasone, and other combinations, for the assessment of efficacy and safety, including patientreported outcomes. ${ }^{25}$

\section{Contributors}

Janssen Research \& Development and PGR designed the study. SLo, BMW, SZU, SS, AC, NJB, AB, AK, RAV, MVM, AM, RZO, HJS, JBl, ECS, AO, JBe, MG, DAS, RL, MS, NC, AJ, DW, JdlR, and PMV gathered the data. SLi, HF, CMU, IK, and TA analysed the data. All authors interpreted the data. SLo wrote the preliminary draft of the manuscript. All authors wrote and reviewed subsequent drafts of the manuscript and approved the final version.

\section{Declaration of interests}

SLo reports consulting for Bristol-Myers Squibb, Celgene, Janssen, Millennium, Novartis, and Onyx and research funding from Janssen for this study. BMW reports consulting for Janssen and Millennium and research funding from Janssen and Prothena. SZU reports consulting for Celgene, Millennium Takeda, Onyx, and Sanofi, speaker's fees for Celgene, Millennium Takeda, and Onyx, and research funding from
Array Biopharma, Celgene, Janssen Oncology, Onyx, Pharmacyclics, and Sanofi. AC reports consulting for Array Biopharma, Celgene, Millennium Takeda, Novartis, and Onyx and research funding from Array Biopharma, Celgene, Janssen Pharmaceuticals, Millennium Takeda, Novartis, Onyx, and Pharmacyclics. NJB reports honoraria from Amgen, Celgene, and Johnson \& Johnson, speaker's fees from Celgene and Johnson \& Johnson, and research funding from Celgene and Johnson \& Johnson. AK reports consulting for Celgene, Onyx, and Bristol-Myers Squibb, speaker's fees from Celgene, Millennium, and Onyx and immediate family ownership of stock and other interests in Celgene and Infiniti. MVM reports consulting for Amgen, Celgene, Janssen, and Takeda, honoraria from Bristol-Myers Squibb, Celgene, and Janssen, and other from Celgene and Janssen. AM reports speaker's fees from Celgene, Millennium, and Onyx. RZO reports consulting for Array BioPharma, Bristol-Myers Squibb, Celgene, FORMA Therapeutics, Janssen, Millennium, and Onyx, research funding from Bristol-Myers Squibb, Celgene, Janssen, Onyx, and Spectrum Pharmaceuticals. HJS reports consulting for Celgene and Janssen and research funding from GlaxoSmithKline and Novartis. JBl reports honoraria from Binding Site, Celgene, Janssen, and Onyx and research funding from Janssen. AO reports consultancy from Janssen, Amgen, and Celgene. JBe reports research funding from Abbvie, Acetylon, Bristol-Myers Squibb,

Calithera, Celgene, Constellation, Epizyme, Karyopharm, Novartis, Onyx, Takeda, and Tragara. MG reports speaker's fees from Takeda. MS reports honoraria from Novartis, Janssen, and Celgene. AJ reports honoraria and consulting for Bristol-Myers Squibb, Celgene, Janssen, Millennium, Onyx, Amgen, Skyline Diagnostics, Karyopharm Therapeutics, and Sanofi, and research funding from Karyopharm. DW reports research funding and personal fees from Celgene and Janssen. PGR reports being a member of the advisory committee for Genmab. SLi is an employee of Genmab. HF, IK, CMU, and TA are employees of Janssen Research \& Development. PMV reports honoraria from Millennium Takeda and Novartis, consulting for Array BioPharma, Bristol-Myers Squibb, Celgene, GlaxoSmithKline, and Oncopeptides, and research funding from Celgene, GlaxoSmithKline, and Oncopeptides. The other authors declare no competing interests.

\section{Acknowledgments}

This study was sponsored by Janssen Research \& Development. We thank the patients who participated in this trial and their families; the study co-investigators, research nurses, and coordinators at each of the clinical sites; representatives of the sponsor who were involved in data gathering and analysis; members of the independent review committee Michael O'Dwyer, Heather Landau, and Shaji Kumar. Medical writing and editorial support was provided by Christopher Jones of MedErgy (Yardley, PA, USA), and was funded by Janssen Global Services.

\section{References}

1 Rollig C, Knop S, Bornhauser M. Multiple myeloma. Lancet 2015; 385: 2197-208.

2 Kumar SK, Rajkumar SV, Dispenzieri A, et al. Improved survival in multiple myeloma and the impact of novel therapies. Blood 2008; 111: $2516-20$

3 Turesson I, Velez R, Kristinsson SY, Landgren O. Patterns of improved survival in patients with multiple myeloma in the twenty-first century: a population-based study. J Clin Oncol 2010; 28: 830-34.

4 Kumar SK, Lee JH, Lahuerta JJ, et al. Risk of progression and survival in multiple myeloma relapsing after therapy with IMiDs and bortezomib: a multicenter international myeloma working group study. Leukemia 2012; 26: 149-57.

5 Kuroda J, Nagoshi H, Shimura Y, Taniwaki M. Elotuzumab and daratumumab: emerging new monoclonal antibodies for multiple myeloma. Expert Rev Anticancer Ther 2013; 13: 1081-88.

6 de Weers M, Tai YT, van der Veer MS, et al. Daratumumab, a novel therapeutic human CD38 monoclonal antibody, induces killing of multiple myeloma and other hematological tumors. J Immunol 2011; 186: 1840-48.

7 Lammerts van Bueren J, Jakobs D, Kaldenhoven N, et al. Direct in vitro comparison of daratumumab with surrogate analogs of CD38 antibodies MOR03087, SAR650984 and Ab79. 56th ASH Annual Meeting and Exposition; San Francisco, CA, USA; Dec 6-9, 2014. Abstract 3474 . 
8 Overdijk MB, Verploegen S, Bogels M, et al. Antibody-mediated phagocytosis contributes to the anti-tumor activity of the therapeutic antibody daratumumab in lymphoma and multiple myeloma. MAbs 2015; 7: 311-21.

9 Jansen JHM, Boross P, Overdijk MB, Lammerts van Bueren JJ, Parren PWHI, Leusen HHW. Daratumumab, a human CD38 antibody induces apoptosis of myeloma tumor cells via FC receptor-mediated crosslinking. Blood 2012; 120: 2974 (abstr)

10 Lokhorst HM, Plesner T, Laubach JP, et al. Targeting CD38 with daratumumab monotherapy in multiple myeloma. $N$ Engl J Med 2015; 373: 1207-19.

11 Durie BGM, Harousseau JL, Miguel JS, et al. International uniform response criteria for multiple myeloma. Leukemia 2006; 20: 1467-73.

12 Rajkumar SV, Harousseau JL, Durie B, et al. Consensus recommendations for the uniform reporting of clinical trials: report of the International Myeloma Workshop Consensus Panel 1. Blood 2011; 117: 4691-95.

13 US Department of Health and Human Services, National Institutes of Health, National Cancer Institute. Common Terminology Criteria for Adverse Events (CTCAE). Version 4.03. http://evs.nci.nih.gov/

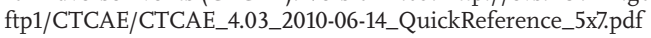
(accessed Nov 5, 2014).

14 Chapuy CI, Nicholson RT, Aguad MD, et al. Resolving the daratumumab interference with blood compatibility testing. Transfusion 2015; published online March 12. DOI:10.1111/trf.13069.

15 Chng WJ, Dispenzieri A, Chim CS, et al. IMWG consensus on risk stratification in multiple myeloma. Leukemia 2014; 28: 269-77.

16 Zonder JA, Mohrbacher AF, Singhal S, et al. A phase 1, multicenter, open-label, dose escalation study of elotuzumab in patients with advanced multiple myeloma. Blood 2012; 120: 552-59.

17 Lonial S, Dimopoulos M, Palumbo A, et al. Elotuzumab therapy for relapsed or refractory multiple myeloma. N Engl J Med 2015; 373: 621-31
18 Harousseau JL, vet-Loiseau H, Attal M, et al. Achievement of at leas very good partial response is a simple and robust prognostic factor in patients with multiple myeloma treated with high-dose therapy: long-term analysis of the IFM 99-02 and 99-04 Trials. J Clin Oncol 2009; 27: 5720-26.

19 Kapoor P, Kumar SK, Dispenzieri A, et al. Importance of achieving stringent complete response after autologous stem-cell transplantation in multiple myeloma. J Clin Oncol 2013; 31: 4529-35.

20 Richardson PG, Siegel DS, Vij R, et al. Pomalidomide alone or in combination with low-dose dexamethasone in relapsed and refractory multiple myeloma: a randomized phase 2 study. Blood 2014; 123: 1826-32.

21 Siegel DS, Martin T, Wang M, et al. A phase 2 study of single-agen carfilzomib (PX-171-003-A1) in patients with relapsed and refractory multiple myeloma. Blood 2012; 120: 2817-25.

22 San Miguel J, Weisel K, Moreau P, et al. Pomalidomide plus low-dose dexamethasone versus high-dose dexamethasone alone for patients with relapsed and refractory multiple myeloma (MM-003): a randomised, open-label, phase 3 trial. Lancet Oncol 2013; 14: 1055-66

23 Plesner T, Arkenau HT, Lokhorst HM, et al. Safety and efficacy of daratumumab with lenalidomide and dexamethasone in relapsed or relapsed, refractory multiple myeloma. Blood 2014; 124: 84 (abstr).

24 Moreau P, Mateos MV, Bladé J, et al. An open-label, multicenter, phase $1 \mathrm{~b}$ study of daratumumab in combination with backbone regimens in patients with multiple myeloma. Blood 2014; 124: 176 (abstr).

25 Laubach JP, Richardson PG. CD38-targeted immunochemotherapy in refractory multiple myeloma: a new horizon. Clin Cancer Res 2015; 21: 2660-62. 